\title{
Rhabdomyolysis and acute renal failure in acute necrotizing encephalopathy with influenza $A$
}

Sirs,

We read with interest the recent paper of Dell and Schulman [1] in this journal. Although influenza myositis has been frequently described, rhabdomyolysis due to influenza virus infection has rarely been reported. Recently, Mizuguchi et al. [2] proposed classifying acute encephalopathy with multifocal, symmetric brain lesions as acute necrotizing encephalopathy (ANE). The etiology of ANE is still unknown, but $24 \%$ of patients have evidence of influenza A infection [2]. We report here a patient with ANE and influenza $\mathrm{A}$ infection, presenting with rhabdomyolysis and acute renal failure.

A previously healthy 5-year-old Japanese boy was admitted to our hospital because of unconsciousness, generalized tonic clonic convulsions, and oliguria. Laboratory studies revealed liver dysfunction (alanine aminotransferase 13,695 U/l, aspartate aminotransferase 5,540 U/l, lactate dehydrogenase $173,822 \mathrm{U} / 1$, total bilirubin $2.1 \mathrm{mg} / \mathrm{dl}$ ), rhabdomyolysis (creatine kinase 8,460 U/l, serum myoglobin $688 \mathrm{ng} / \mathrm{ml}$, urine myoglobin $2,350 \mathrm{ng} / \mathrm{ml}$ ), acute renal failure (urea $80.1 \mathrm{mg} / \mathrm{dl}$, creatinine $3.1 \mathrm{mg} / \mathrm{dl}$, uric acid $21.5 \mathrm{mg} / \mathrm{dl}$, creatinine clearance $12.5 \mathrm{ml} / \mathrm{min}$ per $1.73 \mathrm{~m}^{2}$ ), and disseminated intravascular coagulation. Urinalysis showed $3+$ protein and $3+$ blood, with many red blood cells per high-power field. His cerebrospinal fluid had no pleocytosis. The cranial computed tomography film showed no abnormality on admission.

$\mathrm{He}$ underwent continuous venovenous hemofiltration and plasma exchange. His urine output increased on the $3 \mathrm{rd}$ day after admission, and his level of consciousness gradually increased. The cranial computed tomography film on the 5th day after admission showed bilateral symmetrical thalamotegmental low-density areas (Fig. 1), so he was diagnosed as having ANE. The cranial magnetic resonance film ( $\mathrm{T}_{2}$-weighted image) showed high-intensity areas in the same lesions. Thereafter his condition improved uneventfully and he was discharged on the 40th day after admission without any sequelae. His serum anti-influenza A (H1N1) antibody titer was significantly elevated in the convalescent compared with the acute phase.

To our knowledge, this is the first case of ANE with acute renal failure due to rhabdomyolysis. There are a variety of causes for rhabdomyolysis, including direct trauma to muscle, necrosis from ischemia, and inflammation in muscle from microbiological infections such as in-

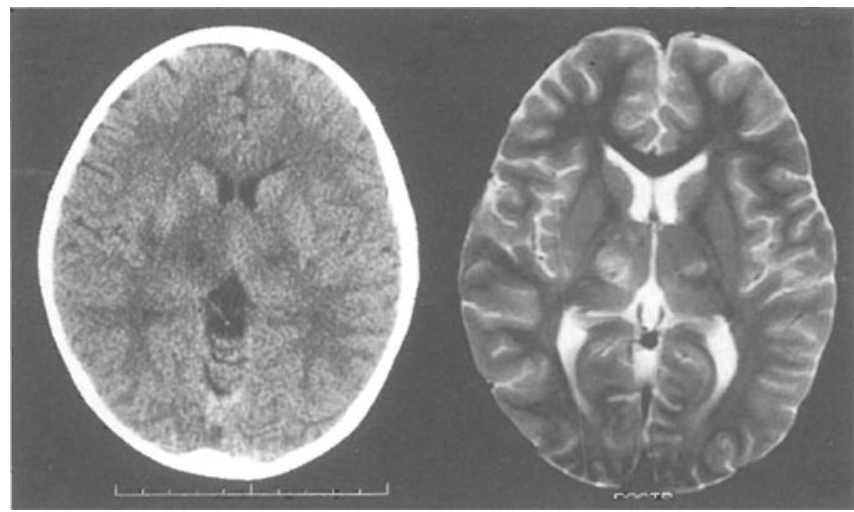

Fig. 1. Left: the precontrast cranial computed tomography film on the 5th day after admission. Right: the cranial magnetic resonance film ( $\mathrm{T}_{2}$-weighted image) on the 10 th day after admission

fluenza virus [3]. Any unconscious patient may suffer from rhabdomyolysis because long motionless periods cause muscle ischemia and pressure necrosis. This pressure muscle necrosis could have induced rhabdomyolysis in our patient. However, his rhabdomyolysis developed soon after the onset of his illness, hence we consider that influenza A infection induced both ANE and myositis, resulting in myoglobinuric acute renal failure.

Toru Watanabe and Yoshihiko Oda

Department of Pediatrics

Niigata City General Hospital

2-6-1 Schichikuyama

Niigata 950, Japan

\section{References}

1. Dell KM, Schulman SL (1997) Rhabdomyolysis and acute renal failure in a child with influenza A infection. Pediatr Nephrol 11: $363-365$

2. Mizuguchi M, Mikkaichi K, Noma S, et al (1995) Acute necrotizing encephalopathy of childhood: a new syndrome presenting with multifocal, symmetric brain lesions. J Neurol Neurosurg Psychiatry 58: $555-561$

3. Brumback RA, Feeback DL. Leech RW (1992) Rhabdomyolysis in childhood. A primer on normal muscle function and selected metabolic myopathies characterized by disordered energy production. Pediatr Clin North Am 39: 821-858 